\title{
High-Harmonic Generation in Solids: Bridging the Gap Between Attosecond Science and Condensed Matter Physics
}

\author{
Giulio Vampa, Chris McDonald, Alec Fraser, and Thomas Brabec
}

(Invited Paper)

\begin{abstract}
We review recent progress in understanding the dominant mechanism driving high-harmonic generation in solids. Three-dimensional two-band single active electron calculations predict that the major emission arises from the recombination of electron-hole pairs upon their creation and acceleration in the laser field, in analogy to atomic high-harmonic generation. The main goal of this study is to review a simple quasi-classical trajectory formalism and use it to better understand the fundamental properties of high-harmonic generation in solids and how they compare to high-harmonic generation in atomic and molecular gases. The simple formalism presents a valuable tool for extending attosecond science from the gas to the condensed matter phase. This is demonstrated by discussing the potential synthesis of attosecond pulses from solids.
\end{abstract}

Index Terms-High harmonic generation (HHG), solids, nonlinear optics, attoseconds.

\section{INTRODUCTION}

$\mathbf{T}$ HE extremely nonlinear interaction of ultrashort intense laser pulses with atomic or molecular targets leads to the production of coherent soft X-Ray radiation [1]. The process, dubbed "high-harmonic generation" (HHG), allowed for new gas-phase spectroscopic methods [2]-[4] and laser sources capable of delivering bursts of light with attosecond duration [5], [6] to be developed.

High-harmonic spectroscopy, for example, has enabled imaging of molecular orbitals [3], [7] and dynamics during a chemical reaction [4]. HHG is also technologically relevant because it provides a well collimated source of spatially and temporally coherent X-Rays, useful for example to perform diffraction experiments [8] and to generate pulses with duration of a few tens of attoseconds [6].

So far, HHG related research has focused on atoms or molecules. Only recently, have high harmonics also been measured from bulk semiconductor crystals [9], [10]. The generation of high harmonics has been made possible by technological progress in intense femtosecond mid-IR and $\mathrm{THz}$ laser sources. In Ref. [9] the authors generate up to the 25th

Manuscript received December 5, 2014; revised February 5, 2015; accepted February 8, 2015.

The authors are with the Department of Physics, University of Ottawa, Ottawa, ON K1N6N5, Canada (e-mail: gvamp015@uottawa.ca; cmcd059@uottawa.ca; alec.fraser@mail.mcgill.ca; thomas.brabec@uottawa.ca).

Color versions of one or more of the figures in this paper are available online at http://ieeexplore.ieee.org.

Digital Object Identifier 10.1109/JSTQE.2015.2402636 harmonic (at $9.5 \mathrm{eV}$ ) of a fundamental wavelength centred at $3.25 \mu \mathrm{m}$ in a $\mathrm{ZnO}$ crystal. The harmonics extend significantly above the minimum bandgap of the material $(3.2 \mathrm{eV})$, suggesting the non-perturbative nonlinearity of these harmonics. The non-perturbative nature becomes apparent from the scaling of the harmonic intensity as a function of the fundamental laser intensity - it does not follow the power law behaviour typical of conventional nonlinear optics [11]. In Ref. [10], a phase-locked THz source with wavelength centred around $10 \mu \mathrm{m}$ was used, and harmonics up to the 20th order were measured. A similar intensity scaling as in Ref. [9] was found.

These experiments have stirred significant theoretical effort to understanding the mechanism driving $\mathrm{HHG}$ in solids [9], [10], [13], [14], [18], [19]-[23]. In solids there are two potential

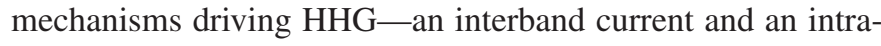
band current. Both are initiated by strong-field excitation of an electron to the conduction band through which an electron-hole pair is formed. Then, the electron and the hole are accelerated by the laser field, thereby gaining momentum and energy and exploring wide portions of the Brillouin Zone (BZ). Whereas the interband current relies on the buildup of polarization between the electron and the hole, the intraband current is driven by the individual motion of electrons and holes in their respective bands. Nonlinearity in the intraband contribution comes from tunnel ionization [12], [22] and from the nonlinear dependence of the band velocity on the electron-hole crystal momentum, which is determined by the laser vector potential [17].

In a recent work, the importance of interband and intraband HHG were examined and it was found that interband HHG is the dominant mechanism [14] for mid-IR excitation laser frequencies. Quasi-classical analysis of interband HHG has revealed a picture that is very similar to $\mathrm{HHG}$ in atomic and molecular gases. Following creation of an electron-hole pair, the laser field first drives the electrons and holes apart. Once the laser field has reversed its direction, the electron and hole are driven back towards each other. Finally, when electron and hole recollide and recombine, a harmonic photon with energy equal to the band energy difference between electron and hole is emitted. Similar models have been used to describe other strong-field interaction processes in solids, such as high-order sideband generation of $\mathrm{THz}$ radiation [15] and electron rescattering at the surface of a nanotip [16].

By comparison, in atomic HHG an electron is first ionized. It is then accelerated — driven away and back to the ion - following 
the sinusoidal evolution of the laser field. The ion is stationary due to its much larger weight. Once it recollides with the ion and recombines to its initial state, a harmonic photon is generated with energy equal to the kinetic energy of the free electron.

In this work we review the method and results derived in Ref. [14], [29] for HHG in solids. Our analysis focuses on interband and recollision based HHG. The paper is structured as follows.

In Section II we review the 3-D theoretical model, which relies on a two band, single active electron approximation. Results from the numerical solution of the full two band equations are discussed. Above the bandgap, interband HHG is dominant which is non-perturbative in nature. For below bandgap harmonics it is shown that HHG is perturbative for field strengths below $\sim 10^{-3}$ atomic units. For higher field strengths non-perturbative mechanisms begin to dominate.

In Section III, the semiclassical model introduced in Ref. [14], [29] is employed to visualize the motion of electron-hole pairs and to explore the similarities and differences of HHG in the gas and condensed matter phase.

In Section IV we investigate the scaling of the cutoff harmonic with the laser field strength and derive an approximated analytical expression based on the semiclassical model.

In Section $\mathrm{V}$ we discuss the influence of dephasing on the harmonic spectrum and on the efficiency of HHG.

Finally, in Section VI we conclude by investigating the potential of HHG in solids for attosecond pulse generation. Our work complements previous analysis on the duration of highharmonic pulses generated via the intraband mechanism [21]. We find that the spectral chirp imprinted by the interband HHG process is substantially larger than in gases and prohibits the generation of transform limited high harmonic pulses. Our analysis predicts pulse durations of slightly above $1 \mathrm{fs}$ in $\mathrm{ZnO}$. By optimizing material parameters, e.g. by using wider bandgap materials, the realization of a sub-fs solid state VUV source should be possible.

\section{THEORETICAL MODELLING}

Our analysis of HHG from $\mathrm{ZnO}$ crystals is based on a 3-D, two-band, single active electron model solid with general band dispersion,

$$
E_{\mathrm{m}}(\mathbf{k})=E_{\mathrm{m}, x}\left(k_{x}\right)+E_{\mathrm{m}, y}\left(k_{y}\right)+E_{\mathrm{m}, z}\left(k_{z}\right)
$$

with $\mathrm{m}=(\mathrm{v}, \mathrm{c})$ for the valence and conduction bands respectively. For each direction in the reciprocal space $(i=x, y, z)$,

$$
\begin{aligned}
& E_{\mathrm{v}, i}\left(k_{i}\right)=\sum_{j=0}^{\infty} \alpha_{\mathrm{v}, i}^{j} \cos \left(j k_{i} a_{i}\right) \\
& E_{\mathrm{c}, i}\left(k_{i}\right)=E_{g}+\sum_{j=0}^{\infty} \alpha_{\mathrm{c}, i}^{j} \cos \left(j k_{i} a_{i}\right)
\end{aligned}
$$

with $a_{i}=2 \pi / \kappa_{i}$ where $\kappa_{i}$ is the reciprocal lattice vector along coordinate $i$. The orientation of the reciprocal lattice of $\mathrm{ZnO}$ (wurtzite structure) is chosen so that $\hat{\mathbf{x}}\|\Gamma-M, \hat{\mathbf{y}}\| \Gamma-K$, and $\hat{\mathbf{z}} \| \Gamma-A$ (optical axis); along these directions [24] $\left(a_{x}, a_{y}, a_{z}\right)=$ $(5.32,4.61,9.83)$ a.u. These parameters are calculated from the reciprocal lattice vectors of the hexagonal wurtzite structure

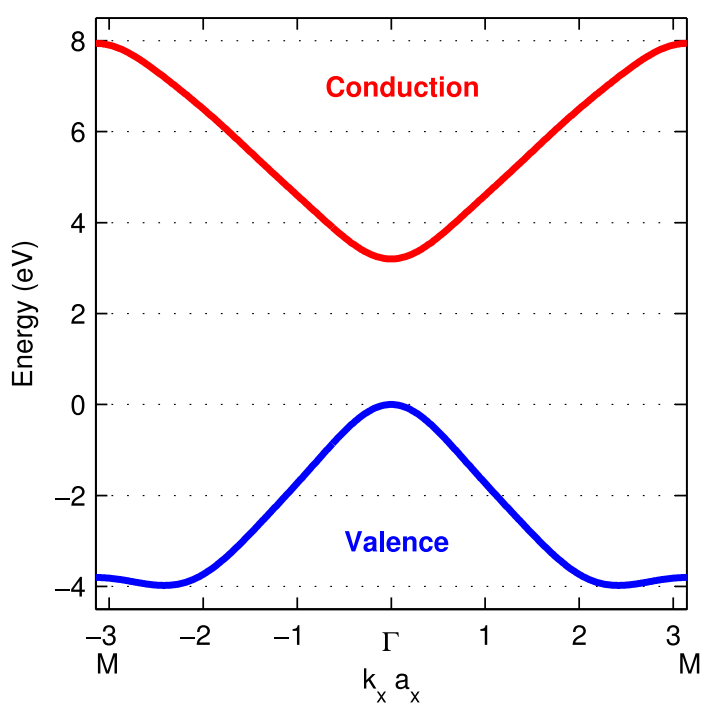

Fig. 1. The first conduction (red line) and the split-off valence (blue line) bands of $\mathrm{ZnO}$ along the $\Gamma-\mathrm{M}$ direction of the $\mathrm{BZ}$ are extracted from Ref. [24]; for the expansion coefficients see Table I.

TABLE I

EXPANSION COEFFICIENTS $\alpha_{\mathrm{m}, i}^{j}(i=x, y, z$ AND $j=0,1 \ldots)$ OF THE NL-EPM BANDS TAKEN FROM REF. [24] FOR THE CRYSTAL FIELD SPLit VALENCE BAND $(m=v)$ AND FOR THE FIRST CONDUCTION BAND $(m=c)$

\begin{tabular}{lcc}
\hline \hline & Valence band & Conduction band \\
\hline$\alpha_{\mathrm{m}, x}^{0}$ & -0.0928 & 0.0898 \\
$\alpha_{\mathrm{m}, x}^{1}$ & 0.0705 & -0.0814 \\
$\alpha_{\mathrm{m}, x}^{2}$ & 0.0200 & -0.0024 \\
$\alpha_{\mathrm{m}, x}^{3}$ & -0.0012 & -0.0048 \\
$\alpha_{\mathrm{m}, x}^{4}$ & 0.0029 & -0.0003 \\
$\alpha_{\mathrm{m}, x}^{5}$ & 0.0006 & -0.0009 \\
$\alpha_{\mathrm{m}, y}^{0}$ & -0.0307 & 0.1147 \\
$\alpha_{\mathrm{m}, y}^{1}$ & 0.0307 & -0.1147 \\
$\alpha_{\mathrm{m}, z}^{0}$ & -0.0059 & 0.0435 \\
$\alpha_{\mathrm{m}, z}^{1}$ & 0.0059 & -0.0435 \\
\hline \hline
\end{tabular}

with lattice constants $a=6.143$ a.u. and $c=9.83$ a.u. Atomic units are used throughout the paper unless otherwise indicated. The bands, calculated with a NL-EPM approach [24], are plotted in Fig. 1. The expansion coefficients $\alpha_{\mathrm{m}, i}^{j}(i=x, y, z$, $j=0,1 \ldots)$ for valence $(\mathrm{m}=\mathrm{v})$ and conduction $(\mathrm{m}=\mathrm{c})$ bands are obtained up to $j=5$ for $\Gamma-M$; the nearest neighbour expansion is used for $\Gamma-K$ and $\Gamma-A$. The resulting coefficients are listed in Table I. The bandgap energy at the $\Gamma$-point is given by $E_{\mathrm{g}}=0.1213$. The calculations have also been performed with bands obtained from DFT in the local density approximation (LDA-ABINIT). Although the spectra show minor differences, the discussion and the conclusions presented here are valid in both cases. In our calculation we use the lowest conduction band and the crystal field split valence band, since its lower effective hole mass results in strongest ionization.

In order to capture the fundamental processes driving $\mathrm{HHG}$ in solids we have confined our analysis to a band energy that is linearly decomposed along an orthogonal coordinate system. This presents an idealization of real semiconductors, such as 
$\mathrm{ZnO}$. We would like to briefly discuss resulting shortcomings of our model and how to correct for them in an improved analysis. From our calculations we find that, at the peak of the pulse, the lateral width of the electron and hole wavepacket extends over $10-40 \%$ of the $\mathrm{BZ}$ for the investigated field strengths, i.e. it remains in the parabolic range along the $\Gamma-K$ and $\Gamma-A$ directions. The band anisotropy in $\mathrm{ZnO}$ is rather small along the $\Gamma-M$ and $\Gamma-K$ directions, justifying the decoupling. There is a stronger asymmetry along the $\Gamma-A$ direction so that the offdiagonal elements of the mass tensor should be considered. Further, when the electron- and hole-wavepacket reach the boundary of the $\mathrm{BZ}$, our orthogonal coordinate system fails to reflect the hexagonal correct symmetry of the $\mathrm{BZ}$ of $\mathrm{ZnO}$. This occurs around the highest field strengths considered in our paper $(F \approx 0.08)$, for which the coordinate system would need to be adapted.

High harmonics are generated by intra- and interband currents [14],

$$
\begin{aligned}
& \mathbf{j}_{\mathrm{ra}}(t)=\sum_{\mathrm{m}=\mathrm{c}, \mathrm{v}} \int_{\mathrm{BZ}} \mathbf{v}_{\mathrm{m}}(\mathbf{k}) n_{m}(\mathbf{k}-\mathbf{A}(t), t) d^{3} \mathbf{k} \\
& \mathbf{j}_{\mathrm{er}}(t)=\frac{d}{d t} \int_{\mathrm{BZ}} \mathbf{p}(\mathbf{k}-\mathbf{A}(t), t) d^{3} \mathbf{k}
\end{aligned}
$$

where the band velocity is defined by $\mathbf{v}_{\mathrm{m}}(\mathbf{k})=\nabla_{\mathbf{k}} E_{\mathrm{m}}(\mathbf{k}), \mathbf{A}(t)$ is the laser vector potential, and $\mathbf{F}(t)=-d \mathbf{A} / d t$ is the laser electric field. In experiments [9], the vacuum field strength $F_{\mathrm{v}}$ is measured. Due to surface reflection (here considered at normal incidence) the peak electric field strength in the material is reduced to $F_{0}=2 F_{\mathrm{v}} /(n+1)=0.69 F_{\mathrm{v}}$, with $n=1.9042$ the material refractive index of $\mathrm{ZnO}$ at the fundamental frequency. The high-harmonic spectrum is obtained from the Fourier transform (FT) of $\mathbf{j}_{\mathrm{t}}=\mathbf{j}_{\mathrm{ra}}+\mathbf{j}_{\mathrm{er}}$, as $\left|\mathrm{FT}\left\{\mathbf{j}_{\mathrm{t}}\right\}\right|^{2}$. The population of band $\mathrm{m}, n_{\mathrm{m}}(\mathbf{k}, t)$, and the interband polarization $\mathbf{p}(\mathbf{k}, t)$ are governed by the semiconductor Bloch equations,

$$
\begin{aligned}
\dot{\pi}(\mathbf{K}, t) & =-\frac{\pi(\mathbf{K}, t)}{T_{2}}-i \Omega(\mathbf{K}, t) w(\mathbf{K}, t) e^{-i S(\mathbf{K}, t)} \\
\dot{n}_{\mathrm{m}}(\mathbf{K}, t) & =i s_{m} \Omega^{*}(\mathbf{K}, t) \pi(\mathbf{K}, t) e^{i S(\mathbf{K}, t)}+\text { c.c. }
\end{aligned}
$$

where multi-electron effects are neglected. Here, $w=n_{\mathrm{v}}-n_{\mathrm{c}}$ is the population difference, $T_{2}$ accounts for dephasing within the relaxation time approximation, $S(\mathbf{K}, t)=\int_{-\infty}^{t} \varepsilon_{\mathrm{g}}(\mathbf{K}+$ $\left.\mathbf{A}\left(t^{\prime}\right)\right) d t^{\prime}$ is the classical action, $\varepsilon_{\mathrm{g}}=E_{\mathrm{c}}-E_{\mathrm{v}}$ is the bandgap between conduction and valence bands, $\Omega(\mathbf{K}, t)=\mathbf{F}(t) \mathbf{d}(\mathbf{K}+$ $\mathbf{A}(t))$ is the Rabi frequency, and $s_{m}=-1,1$ for $\mathrm{m}=\mathrm{v}, \mathrm{c}$, respectively. Further, the crystal momentum $\mathrm{k}$ has been transformed into a frame moving with the vector potential, $\mathbf{K}=$ $\mathbf{k}-\mathbf{A}(t)$. Finally, $\pi(\mathbf{K}, t)$ is related to the polarization by

$$
\mathbf{p}(\mathbf{K}, t)=\mathbf{d}(\mathbf{K}+\mathbf{A}(t)) \pi(\mathbf{K}, t) e^{i S(\mathbf{K}, t)}+\text { c.c. }
$$

where $\mathbf{d}(\mathbf{k})=i \int d^{3} \mathbf{x} u_{\mathrm{v}, \mathbf{k}}^{*}(\mathbf{x}) \nabla_{\mathbf{k}} u_{\mathrm{c}, \mathbf{k}}(\mathbf{x})$ is the transition dipole moment, with $u_{\mathrm{m}, \mathrm{k}}$ the periodic part of the Bloch function. The $\mathbf{k}$-dependent dipole $\mathbf{d}(\mathbf{k})$ is defined as in the supplementary material of Ref. [14]. Initially all electrons are in the valence band. For a more detailed derivation see Ref. [14].

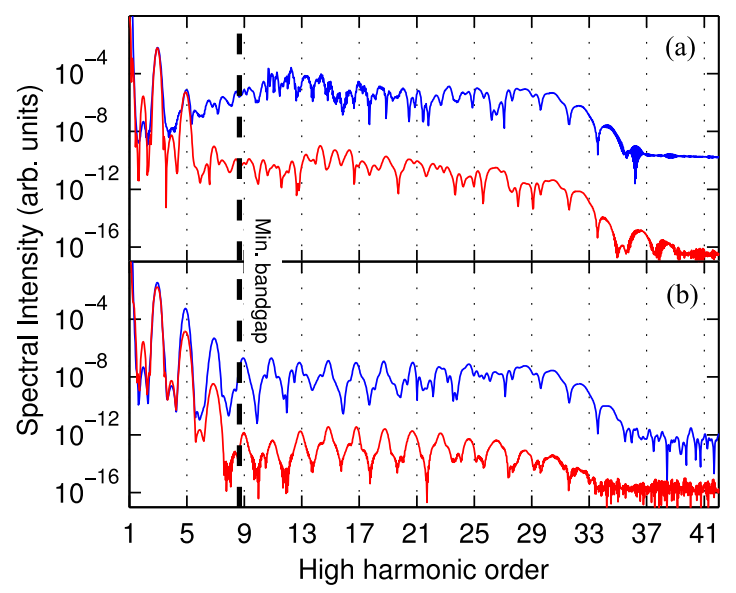

Fig. 2. Harmonic spectrum for interband $\left(\left|F T\left\{\mathbf{j}_{\mathrm{er}}\right\}\right|^{2}\right.$, blue line) and intraband ( $\left|F T\left\{\mathbf{j}_{\mathrm{ra}}\right\}\right|^{2}$, red line) currents for a field strength $F_{0}=0.0045$ a.u.; we use a temporal Gaussian envelope with a FWHM of ten cycles and cosine carrier with frequency $\omega=0.014$ a.u., corresponding to a laser period $T_{0}=2 \pi / \omega=10.9$ fs. The dephasing time is (a) $T_{2}=\infty$ and (b) $T_{2}=T_{0} / 4$. The laser polarization is parallel to the $\Gamma-\mathrm{M}$ direction of the reciprocal space of a wurtzite $\mathrm{ZnO}$ crystal. The dashed black vertical line represents the minimum bandgap at the center of the BZ.

\section{A. Discussion of High Harmonic Spectra}

Fig. 2 shows the high harmonic spectrum of the intraband (red) and interband (blue) curves obtained from $\mathrm{ZnO}$ for two dephasing times $T_{2}$ at a field strength of $F_{0}=0.0045$ a.u. $\left(0.24 \mathrm{~V} / \AA\right.$ or $\left.0.7 \mathrm{TW} / \mathrm{cm}^{2}\right)$ and frequency $\omega=0.014$ a.u. $(\lambda=$ $3.26 \mu \mathrm{m})$. Laser polarization is along the $\Gamma-\mathrm{M}$ direction of the reciprocal space.

Interband harmonics dominate intraband emission for both dephasing times. We have performed calculations in a range of field strengths $0.002 \leq F_{0} \leq 0.01$. Interband HHG is always the dominant mechanism for above bandgap harmonics. For below bandgap harmonics, dominance of interband and intraband HHG varies with intensity.

A comparison of the harmonic spectra in Fig. 2(a) and (b) reveals a strong sensitivity of HHG to dephasing. Fig. 2(a) shows the spectrum in the absence of dephasing; contrary to experimental findings, no clear harmonic structure is observed. A peak-to-valley contrast comparable to that achieved in experiments [9] is observed only for dephasing times shorter than a laser half cycle. The role of dephasing has been explained in Ref. [14] in the following way. Interband emission is understood in terms of a three step model: an electron-hole pair is created by tunnel ionization, driven first apart by the laser field, and then driven back towards each other, once the laser field changes sign, resulting in recollision and recombination of the electron-hole pair; after the first re-encounter the electron-hole pair is again driven apart and then towards each other, so that multiple recollisions can occur at varying time intervals. The interference of these aperiodic contributions to harmonic emission washes out the harmonic structure and a continuum-like spectrum is produced [see Fig. 2(a)]. For dephasing times shorter than $T_{0} / 2$ the coherence between electron and hole is lost for recollisions higher than the first, resulting in a suppression of harmonic emission from higher order returns. 


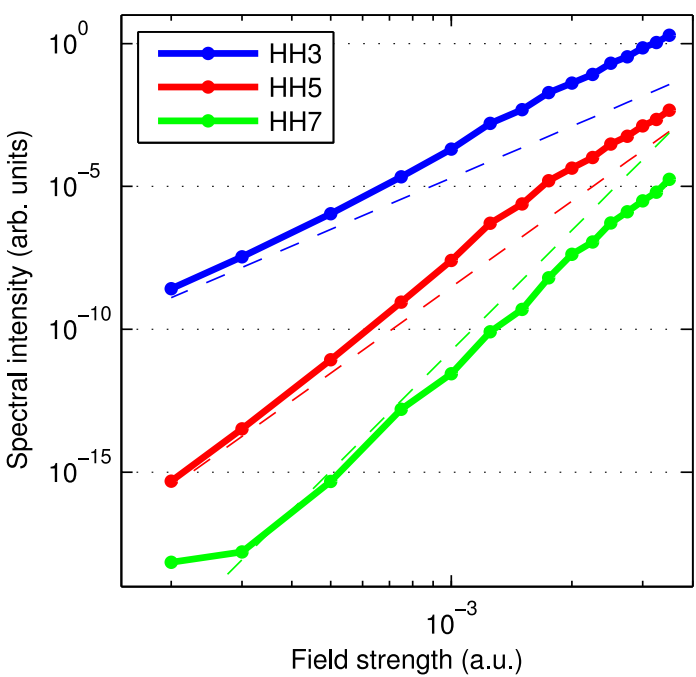

Fig. 3. Total high-harmonic intensity $\left(\left|\mathrm{FT}\left\{\mathbf{j}_{\mathrm{t}}\right\}\right|^{2}\right)$ as a function of fundamental field strength for below bandgap harmonics; pulse parameters are the same as in Fig. 2. The solid lines with round markers represent the simulated spectrum, whereas the dashed lines correspond to the perturbative scaling of each order as given by (6).

\section{B. From Perturbative to Non-Perturbative Regimes}

Harmonics above the minimum bandgap are driven by mechanisms that are usually non-perturbative in nature. The intensity of non-perturbative harmonics is, among other factors, determined by strong-field ionization [25]. As a result, the harmonic yield as a function of driving laser intensity does not follow a power law

$$
I_{N \omega} \propto I_{\omega}^{N}
$$

where $N$ is the order of the harmonic. Below the bandgap, it is not so clear whether perturbative or non-perturbative mechanisms dominate HHG in solids. Fig. 3 shows the intensity of below-bandgap harmonics generated by the total current $\mathbf{j}_{\mathrm{t}}$ as a function of the fundamental field strength (solid lines with markers). The expected perturbative scaling is traced by the dashed lines in Fig. 3. Only for $F_{0}<10^{-3}$ a.u. are harmonics generated perturbatively. At higher intensities below-bandgap harmonics become non-perturbative. In atomic HHG, several mechanisms to generate these harmonics have been proposed in the literature [26], and have been experimentally verified [27]. It is possible that similar mechanisms are at play in HHG from solids as well.

\section{SEMICLASSICAL DESCRIPTION OF INTERBAND HHG}

In the limit of low ionization, $\mathbf{w}(\mathbf{t})=\mathbf{n}_{\mathbf{v}}(\mathbf{t})-\mathbf{n}_{\mathbf{c}}(\mathbf{t}) \approx \mathbf{1}$, the intra- and interband currents have the analytical solution derived in Ref. [14], from which a simple semiclassical interpretation of $\mathrm{HHG}$ can be derived. In this section we review this model. We focus our discussion on interband HHG which was found above to be the dominant channel. The reliability of the semiclassical trajectory approach will be demonstrated by comparison with numerical simulations.

By integrating Eqs. (4) and inserting the polarization into Eq. (3b), the interband current can be expressed in terms of integrals,

$$
\begin{array}{r}
\mathbf{j}_{\mathrm{er}}(\omega)=\omega \int_{\mathrm{BZ}} d^{3} \mathbf{k} \mathbf{d}(\mathbf{k}) \int_{-\infty}^{\infty} d t e^{-i \omega t} \int_{-\infty}^{t} d t^{\prime} F\left(t^{\prime}\right) d^{*}\left(\boldsymbol{\kappa}_{t^{\prime}}\right) \\
\times e^{-i S\left(\mathbf{k}, t^{\prime}, t\right)-\left(t-t^{\prime}\right) / T_{2}}+\text { c.c. }
\end{array}
$$

where $\boldsymbol{\kappa}_{t^{\prime}}=\mathbf{k}+\mathbf{A}\left(t^{\prime}\right)-\mathbf{A}(t)$. Further, we have transformed the crystal momentum back to the initial frame $\mathbf{k}=\mathbf{K}+\mathbf{A}(t)$, and $S\left(\mathbf{k}, t^{\prime}, t\right)=\int_{t^{\prime}}^{t} \varepsilon_{\mathrm{g}}\left(\boldsymbol{\kappa}_{\tau}\right) d \tau$. The strongest contribution to the integrals come from those points where the phase is stationary, i.e. where the first derivative of the phase is zero. As a result, the three integrals with respect to $t, t^{\prime}$, and $\mathbf{k}$ yield the following three conditions defining the stationary phase points,

$$
\begin{aligned}
\nabla_{\mathbf{k}} S & =\int_{t^{\prime}}^{t} \mathbf{v}\left(\mathbf{k}-\mathbf{A}(t)+\mathbf{A}\left(t^{\prime \prime}\right)\right) d t^{\prime \prime}=0 \\
\frac{d S}{d t^{\prime}} & =\varepsilon_{\mathrm{g}}\left(\mathbf{k}-\mathbf{A}(t)+\mathbf{A}\left(t^{\prime}\right)\right)-\frac{i}{T_{2}}=0 \\
\frac{d S}{d t} & =\varepsilon_{\mathrm{g}}(\mathbf{k})-\Omega+\frac{i}{T_{2}}=0
\end{aligned}
$$

where $\mathbf{v}(\mathbf{k})=\nabla_{\mathbf{k}} \varepsilon_{\mathrm{g}}(\mathbf{k})=\mathbf{v}_{\mathrm{c}}-\mathbf{v}_{\mathrm{v}}$ is the difference of the velocities of the electron and the hole in their respective bands. Further, $\mathbf{x}_{\mathrm{c}, \mathrm{v}}\left(t^{\prime}, t\right)=\int_{t^{\prime}}^{t} d t^{\prime \prime} \mathbf{v}_{\mathrm{c}, \mathrm{v}}$ is the space excursion of electron and hole.

Even in the absence of dephasing, solution of the above equations requires complex stationary phase (saddle) points. The imaginary components capture the essential quantum aspects of the process, such as the tunnelling of the electron from the valence to the conduction bands [28]. After the electron-hole pair is born, its dynamics can be understood by a purely classical description, which corresponds to the real parts of the saddle point solutions. In order to be able to focus on the real part, dephasing is also neglected, i.e. $T_{2}=\infty$.

The first of the three equations, $\nabla_{\mathbf{k}} S=\Delta x_{c}-\Delta x_{v}=0$, states that the total distance travelled by the electron equals that travelled by the hole. Since they are born at the same position, the condition implies that high harmonics are emitted only upon re-encounter of electron and hole.

Eq. (8b) also has a classical interpretation. First, it defines the birth time at which the electron-hole pair is formed by tunnel ionization. Second, it determines the quasi-classical evolution of electron and hole crystal momentum in the laser field. It is easily proven [29] that for direct bandgap materials, for which the minimum bandgap is at the $\Gamma$-point, the solution of the equation is

$$
\mathbf{k}\left(t, t^{\prime}\right)=\mathbf{A}(t)-\mathbf{A}\left(t^{\prime}\right)
$$

which is the classical equation of motion for electron-hole pairs in a lattice.

Finally, Eq. (8c) ensures conservation of energy: the electron recombines with the hole and emits one photon with energy equal to the energy bandgap at the crystal momentum of recombination.

Thus, in essence HHG from solids is driven by a process similar to the three-step model of atomic HHG: an electronhole pair is created at time of birth $t^{\prime}$ (step one); the electron 

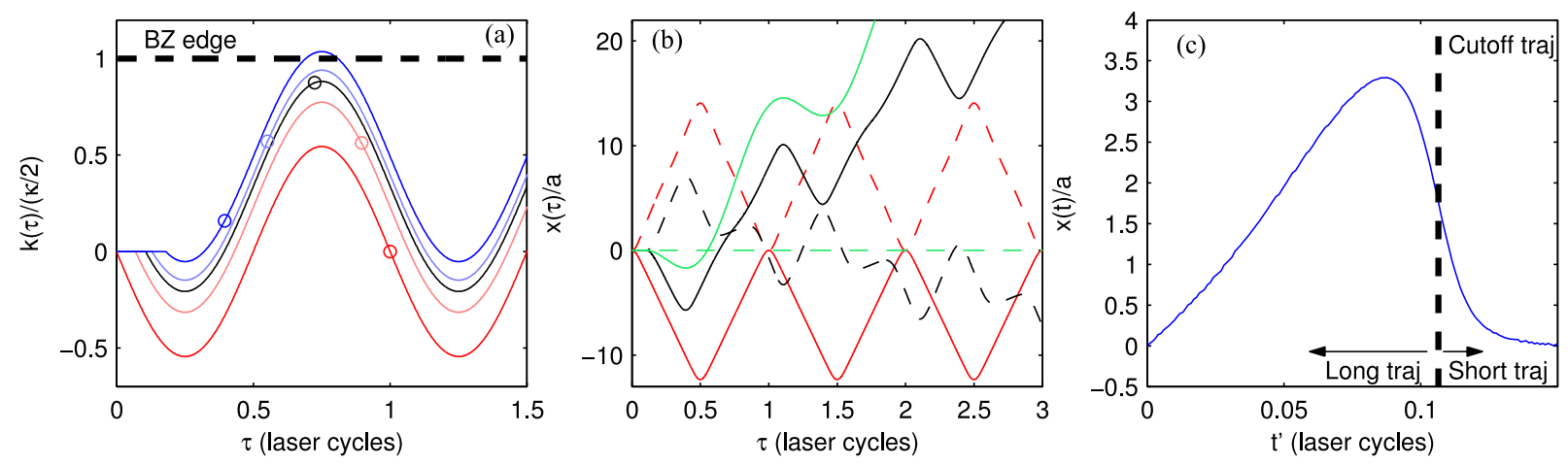

Fig. 4. Semiclassical calculation of electron and hole trajectories for a continuous wave laser with peak field strength $F_{0}=0.0045$ a.u. (a) Crystal momentum obtained with Eq. (9) for electron-hole pairs created at different times. Red (blue) lines correspond to long (short) trajectories for various birth times. The black line represents the cutoff trajectory, for which electron-hole pairs recollide with maximum momentum. The momentum of recollision is marked for each trajectory by the empty circles. The edge of the BZ is marked by the black dashed line; at the chosen $F_{0}$ some trajectories go beyond the first BZ. (b) Position of the electron (solid lines) and the hole (dashed lines) following their creation, for pairs born at the peak of the field (red lines) and for the cutoff trajectory (black lines). The green lines are the atomic trajectories for an electron-hole pair born at the same time as the cutoff trajectory in $\mathrm{ZnO}$. (c) Position of the electron-hole pair at the time of recollision in units of lattice cells. The cutoff trajectory separates long trajectories (smaller birth times) to the short trajectories (longer birth times). Band parameters are given in Table I.

and hole are accelerated by the field in opposite directions (step two); when the field changes sign they are driven back towards their point of origin and eventually re-encounter each other at time $t$ with non-zero crystal momentum (step 3 ). The crystal momentum determines the bandgap energy difference between electron and hole and therewith the energy of the photon that is emitted upon recombination.

We solve the saddle point equations in the classical approximation as follows. For each birth time $t^{\prime}$ an electron and a hole are propagated with velocity $\mathbf{v}\left[\mathbf{k}\left(\tau, t^{\prime}\right)\right]=\mathbf{v}\left[\mathbf{A}(\tau)-\mathbf{A}\left(t^{\prime}\right)\right]$ until time $t$ when they re-encounter with crystal momentum $\mathbf{k}=\mathbf{k}\left(t, t^{\prime}\right)=A(t)-A\left(t^{\prime}\right)$. Equations (8a) and (8b) are thus satisfied. The two stationary times define a classical trajectory, which is associated with a high harmonic photon energy $\varepsilon_{g}\left(\mathbf{k}\left(t, t^{\prime}\right)\right)$ via Eq. (8c).

Fig. 4(a) shows the evolution of $\mathbf{k}\left(\tau, t^{\prime}\right)$ with time $\tau$ for electron-hole pairs created at selected times after the peak of the electric field, which is at $\tau=0$; the trajectories are plotted starting at their respective birth times. The black line represents the trajectory that recollides with highest momentum generating the cutoff harmonic; the red (blue) lines correspond to trajectories that recollide after (before) the cutoff trajectory, and are called "long trajectories" ("short trajectories"). Every trajectory is born with zero momentum and subsequently evolves as dictated by Eq. (9). Electron and hole velocity are determined by the gradient with respect to crystal momentum of the respective bands. As conduction and valence band are different, this results in a different dynamics of electrons and holes. The empty circles indicate the recollision time at which electron and hole re-encounter.

Fig. 4(b) shows the position of electron (solid line) and hole (dashed line) in real space, obtained by integrating over electron and hole velocity. The black and red curves correspond to the same-coloured graphs in Fig. 4(a) representing the cutoff trajectory and an electron-hole pair born at the field maximum $\tau=0$, respectively. For comparison, the trajectory expected from an atomic system driven at the same field strength and born at the same time as the cutoff trajectory is plotted as the green lines in Fig. 4(b). Connection between the atomic gas and the solid state picture can be established by Taylor expanding the bands about the $\Gamma$-point, where they depend quadratically on the crystal momentum. In this limit the band velocity $\mathbf{v}=\nabla_{\mathbf{k}} \varepsilon_{\mathrm{g}} \propto \mathbf{k}\left(t^{\prime}, t\right)$, as in the atomic case. We set $\mathbf{v}=\mathbf{k}\left(t^{\prime}, t\right)$ to simulate the latter. Then, by associating $E_{\mathrm{g}}$ with the atomic ionization potential, quasi-classical equations are obtained that are identical with the atomic case.

Several features of solid HHG can be explained with the semiclassical model. For the following analysis a continuous wave laser field is used.

There are several similarities between HHG in atomic gases and in solids. Similar to the atomic case, trajectories born after the peak of the field acquire a drift velocity. Therefore electron and hole can re-encounter each other only a limited number of times. The time of the first recollision for each trajectory is marked by the open circles in panel (a). As the birth time increases (from the dark red to the light red lines), the momentum of recollision increases, up to the black line which corresponds to the trajectory recolliding with maximum momentum. Because the bandgap monotonically increases with momentum, the high harmonic photon energy also increases. These trajectories correspond to the so-called "long trajectories" found in atomic HHG. As the birth time increases further, the momentum of recollision decreases (from the light blue to the dark blue lines). The highharmonic photon energy also decreases. These trajectories correspond to the "short trajectories" of atomic HHG.

There are also key features where HHG in gases and in solids exhibits different behaviour. First, in contrast to the atomic case, where the mass of the hole is infinite, the hole also moves for its mass is comparable to that of the electron. Because the motion of electron and hole are different from each other due to the differences in energy bands, re-encounter in general occurs offset from their place of birth. This is shown in Fig. 4(c), where the position of recollision is plotted as a function of the birth time. For example, recollision of the cutoff trajectory occurs 


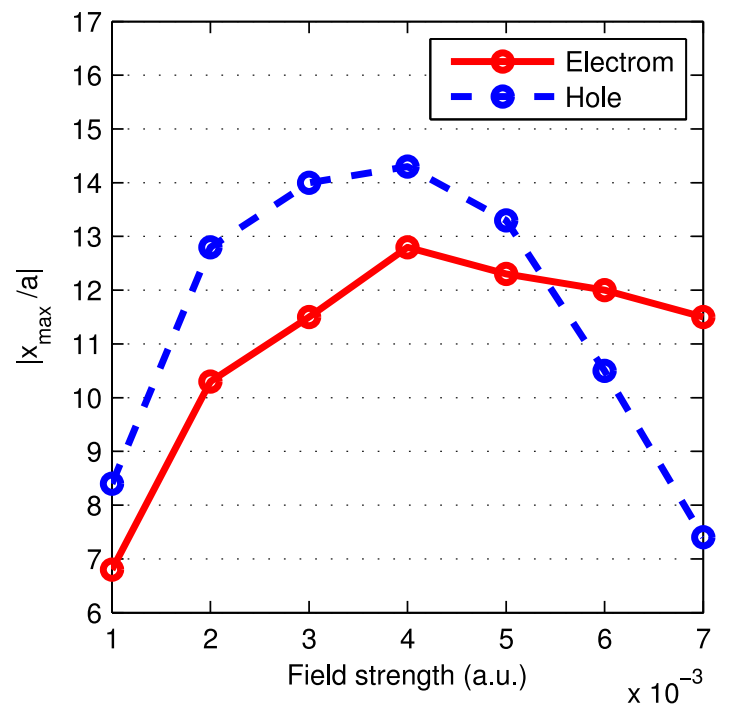

Fig. 5. Maximum excursion before recollision for electron and holes in units of lattice cells as a function of field strength; maximum excursion is obtained for electron-hole pair born at the peak of the laser field; continuous wave laser field with frequency $\omega=0.014$ a.u. is used.

$\sim 1.7$ lattice cells away from its origin. This is not possible in gases, as the atomic nucleus does not move.

Furthermore, as discussed above, only over parts of the bandgap (close to the $\Gamma$-point) where the band velocity is linearly related to the crystal momentum, the atomic picture is retrieved. In general, for higher field strengths parts of the BZ are explored where the bands are anharmonic functions of the crystal momentum $\mathbf{k}\left(t, t^{\prime}\right)$. This results in a different evolution of trajectories in gases and in solids, as seen from a comparison of the green (gas) and black (solid) solid lines in Fig. 4(b).

Finally, in atomic HHG the kinetic energy is an unbounded function of the electron velocity. As a result, electron kinetic energy and excursion can grow indefinitely with $F_{0} / \omega$ and with $F_{0} / \omega^{2}$, respectively. In a solid, the electron-hole velocity is determined by the gradient of the bandgap; as a result, both velocity and excursion amplitude are capped. In strong laser fields the band structure, and therefore the velocity, can be modified by mixing with higher bands, which is not accounted for in our two-band analysis. Fig. 5 shows the maximum excursions before recollision occurs as a function of $F_{0}$. These are reached by electrons born at the peak of the field and recolliding at the end of the cycle. As the field is increased above $F_{0}=0.004$ a.u., the excursion plateaus at $\sim 13$ lattice cells, then decreases for even higher field strengths. Other trajectories travel shorter distances. For example, the excursion amplitude is $\sim 7$ lattice cells at $F_{0}=0.004$ a.u. for the cutoff trajectory.

In Fig. 6 the validity of the quasi-classical trajectory analysis is verified by comparing it to numerical results for two different field strengths. The same parameters are used as in Fig. 2(b). The white line represents the quasi-classical high-harmonic photon energy plotted versus recollision time for all trajectories born between the peak and the next zero of the field. Only recollisions within one optical cycle from the moment of ionization are considered. Short and long trajectories are clearly identified in
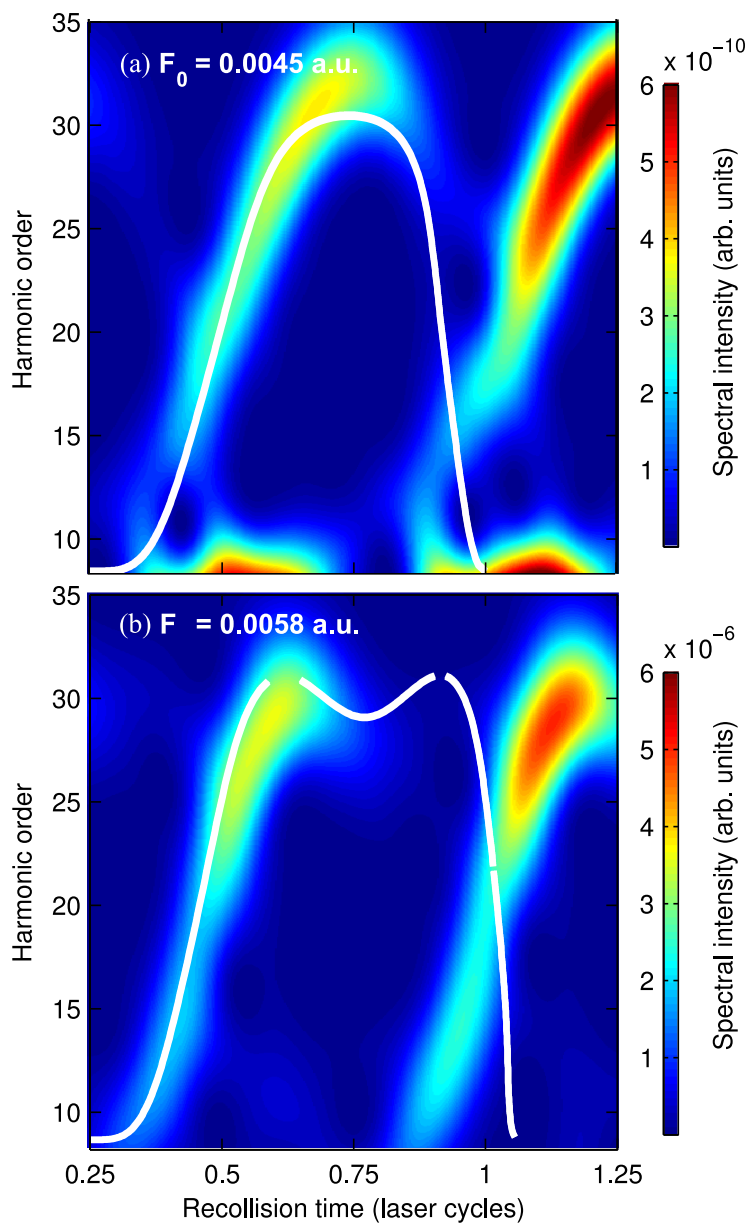

Fig. 6. High harmonic photon energy as a function of time. The semiclassical prediction (white line) agrees with the simulation. Recollision at the next half cycle leads to further identical emission in the simulated data. The simulation is a result of a windowed Fourier transform of the interband current obtained for Fig. 2(b), with dephasing time $T_{2}=T_{0} / 4$. The window is a 0.34 cycles wide Blackman function.

Fig. 6(a) as the rising and falling edges of the curve. The emitted harmonic order as a function of time is extracted from the numerical results in the following way. We performed a windowed Fourier transform of the interband current, where a 0.34 cycles wide Blackman window is scanned across one optical cycle. The time-dependent spectral intensity is color coded in Fig. 6. Because the HHG process repeats itself at the next half cycle, a replica of the time-dependent spectrum is also observed. At the higher field strength of Fig. 6(b) the trajectories are distorted. This behaviour is a result of the non-parabolic and bounded band dispersion that differentiates solid HHG from atomic HHG. It is thoroughly investigated in Ref. [29]. Finally, the good agreement between quasi-classical and numerical results suggests that within the two-band approximation the field-free band structure can be used to approximate the electron and hole trajectories that lead to high-harmonic emission.

\section{Cutoff Scaling LaW}

The maximum high-harmonic produced by the recolliding electron-hole pair can be extracted from the windowed Fourier 


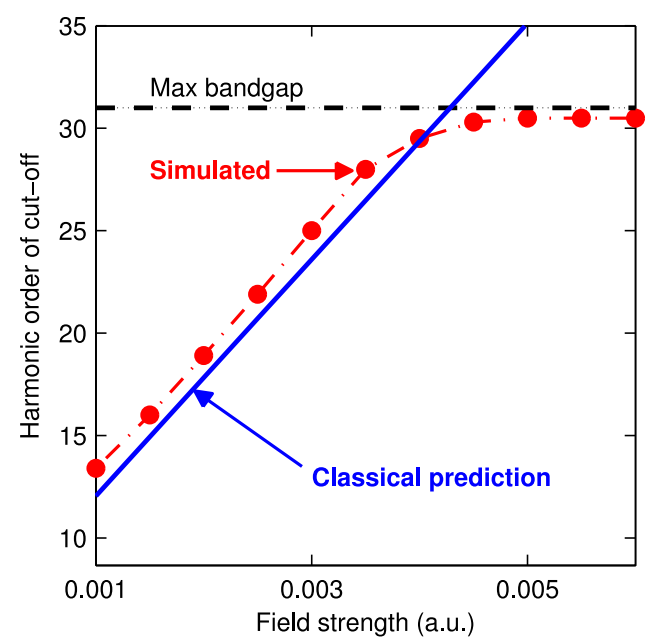

Fig. 7. The cutoff extracted from a windowed Fourier transform of the simulated time-dependent interband dipole (red dots with dashed line) grows linearly with the field strength, and is in good agreement with the semiclassical prediction based on the trajectory analysis (blue line). The maximum bandgap is marked by the black dashed line. Laser and band parameters are the same as in Section II.

transform of Fig. 6 for different field strengths. The cutoff, reported as the red dots with dashed lines in Fig. 7, scales linearly with the field strength, in agreement with what was reported experimentally [9]. The linear scaling is a direct consequence of the linearity of the bandgap with respect to momentum. According to Eq. (8c), the cutoff harmonic is emitted by the trajectory that recollides with highest momentum,

$$
\Omega_{c}=\varepsilon_{\mathrm{g}}\left(\mathbf{k}_{c}\right)
$$

with the momentum of the cutoff trajectory defined by the birth time $t_{c}^{\prime}$ and recollision time $t_{c}$,

$$
k_{c}=\frac{F_{0}}{\omega}\left[\sin \left(\omega t_{c}^{\prime}\right)-\sin \left(\omega t_{c}\right)\right] .
$$

Since momentum scales linearly with field strength (Eq. 9), a bandgap that is linear in momentum yields a cutoff harmonic that increases linearly with field strength. In atomic HHG, where the energy is a quadratic function of momentum, the cutoff scales linearly with the field intensity, as well known from gas phase experiments.

The semiclassical model is particularly useful because it allows for the derivation of an approximate analytical formula for the cutoff. By expanding Eq. (10) up to first order in momentum around the half-point of the $\mathrm{BZ}, k=k_{x} / 4$, the cutoff harmonic can be expressed as:

$$
\Omega_{c} \simeq \varepsilon_{g}\left(\frac{\kappa_{x}}{4}\right)+\left.\frac{d \varepsilon_{g}\left(k_{c}\right)}{d k_{c}}\right|_{k_{c}=\kappa_{x} / 4}\left(k_{c}-\frac{\kappa_{x}}{4}\right) .
$$

The blue line of Fig. 7 is calculated with the above formula, for the band parameters reported in Table I. The cutoff trajectory is found with the semiclassical model described in the previous section.

A detailed discussion of cutoff is reported in Ref. [29].

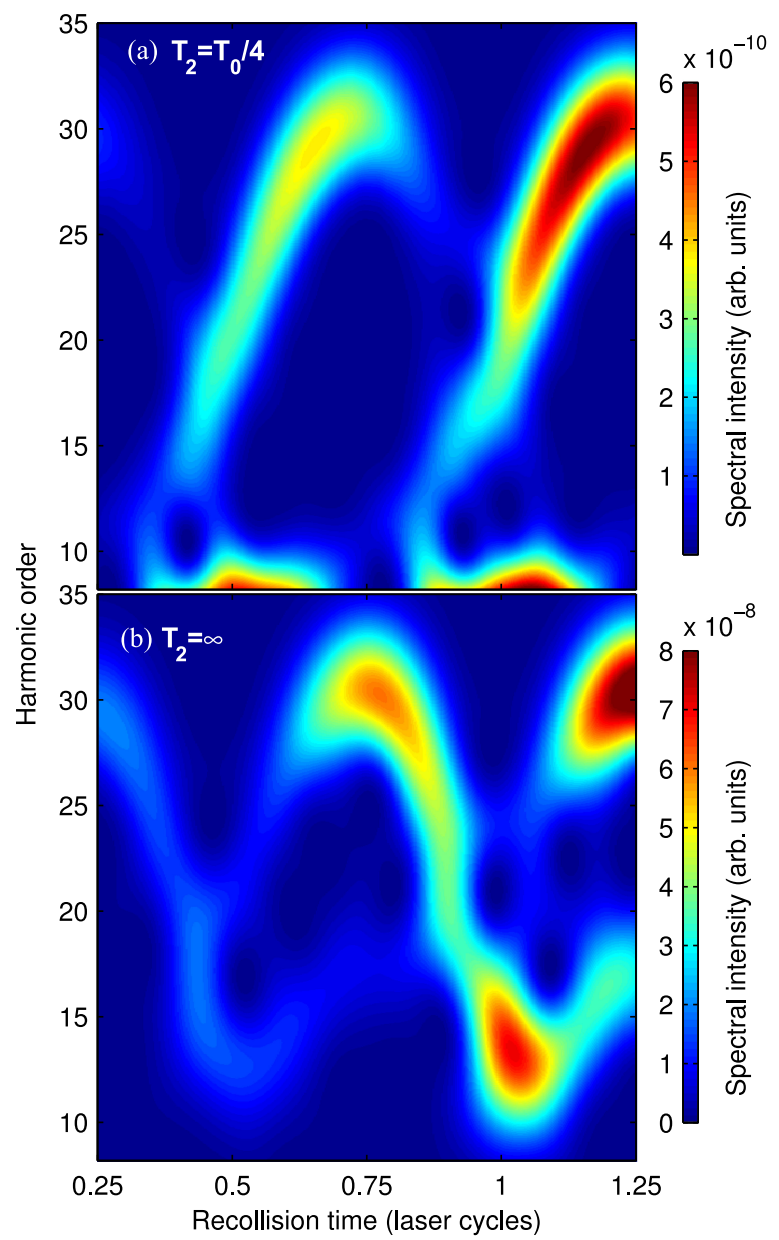

Fig. 8. Time frequency analysis of the interband current for (a) $T_{2}=T_{0} / 4$ and (b) $T_{2}=\infty$. In (b) the long trajectories are more intense than in (a). Furthermore, multiple recollisions are allowed in (b) and result in spectral interferences that wash out the clear odd-harmonic structure. They are clearly visible below $\mathrm{HH} 25$.

\section{EFFECTS OF DEPHASING ON HHG}

Although there exist various mechanisms affecting electronhole recombination, such as quantum diffusion (wavepacket spreading), dephasing appears to be the most prominent one. Dephasing of the interband polarization arises from scattering of the electron and the hole with other particles, which destroys phase coherence between electrons and holes; it enters the interband current via the term

$$
e^{-\left(t-t^{\prime}\right) / T_{2}}
$$

As dephasing is exponentially sensitive to the excursion time, it strongly filters short trajectories with respect to longer trajectories. Fig. 8 shows the high-harmonic intensity with subcycle resolution for two dephasing times: (a) $T_{2}=T_{0} / 4$ and (b) $T_{2}=\infty$. The spectra are again obtained with a windowed Fourier transform; the same parameters are used as those in Fig. 6. In panel (a) recollision from the short trajectories is apparent, whereas the long trajectories are strongly suppressed. In the absence of dephasing (panel b), however, long trajectory components and multiple returns are allowed and they interfere 


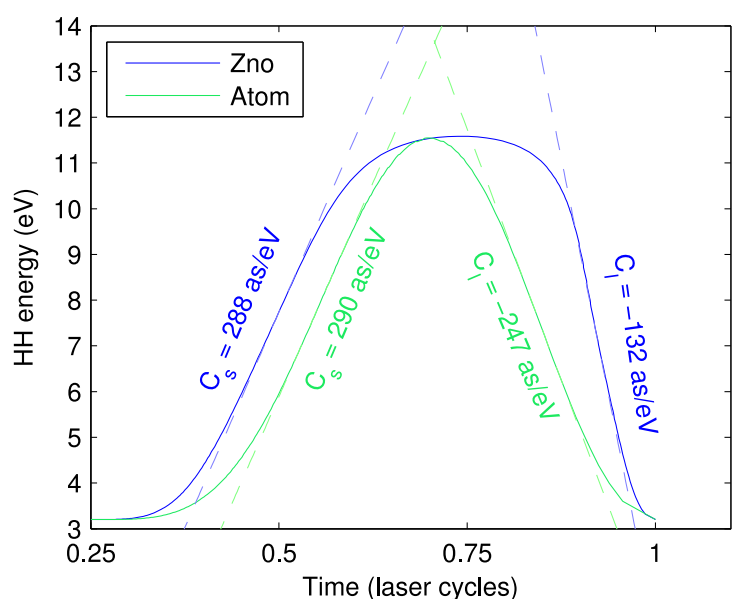

(a)

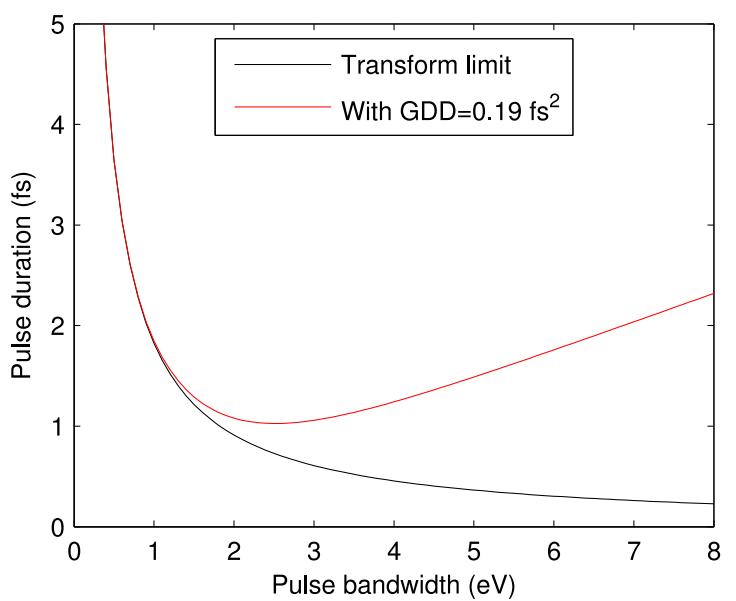

(b)

Fig. 9. (a) High harmonic photon energy as a function of the emission time for $\mathrm{ZnO}$ at $F_{0}=0.0045$ a.u. (blue solid line) and for an atomic system with the same bandgap as $\mathrm{ZnO}$ at $F_{0}=0.0087$ a.u. (green solid line). Laser frequency is $\omega=0.0141$ a.u. The slope of the curves determines the chirp of the high-harmonic pulses. The dashed lines are the derivatives taken at $7.5 \mathrm{eV}$ for the long $\left(C_{l}\right)$ and short $\left(C_{s}\right)$ trajectories. The values of the chirps are reported along the lines. (b) Variation of the pulse duration as a function of the pulse bandwidth. The short trajectory chirp equivalent to GDD $=0.19$ fs ${ }^{2}$ broadens the pulse (red line) with respect to its transform limit (black line). For this GDD the shortest pulse (1.02 fs) is obtained for a $2.5 \mathrm{eV}$ bandwidth.

with the short branch resulting in aperiodic high-harmonic emission. The spectral signature of aperiodic emission is a loss of contrast between adjacent harmonic orders, as in Fig. 2(a). Note also a difference of about two orders of magnitude in the harmonic intensities between HHG with and without dephasing. Furthermore, the exponential dependence in Eq. (13) also results in an exponential dependence of harmonic intensity on the wavelength of the driving field. This has been confirmed by a strong decrease in harmonic intensity for increasing midIR wavelengths found in recent simulations [14]. If experiments confirm this behaviour, dephasing will become measurable. As a result, HHG in solids presents a novel way to measure collisions and dephasing in strongly excited condensed matter systems.

\section{DURATION OF High-HARMONIC PULSES}

One of the most important applications of atomic HHG is the possibility to synthesize XUV laser pulses with a duration of a fraction of a cycle of the fundamental; that is, several tens of attoseconds. The shortest pulse ever generated was 67 as ( 1 as $=$ $10^{-18} \mathrm{~s}$ ) [6] in duration. In solids, as long as recollision-based HHG dominates, also sub-cycle bursts of harmonic radiation are created. Therefore it is in principle possible to isolate (gate) a single pulse much shorter than the cycle of the fundamental field. In practice, a suitable scheme for gating a single recollision is yet to be found. Several gating techniques in atomic gases take advantage of the strong dependence of harmonic emission on the ellipticity of the fundamental laser pulse [30]. The weak ellipticity dependence of high-harmonics from solids measured in Ref. [9] makes the use of these techniques challenging. In solids, a recently developed technique appears to be better suited: it relies on the temporal rotation of the fundamental wavefront to spatially separate each burst in the far-field [31].

It is interesting to consider how short such an isolated pulse in principle can be. Short pulses require wide bandwidths and nearly flat spectral phases (small chirps). The bandgap of $\mathrm{ZnO}$ allows generation of 20 harmonic orders at $\omega=0.014$ a.u., corresponding to $7.6 \mathrm{eV}$ bandwidth. If we assume a transformlimited Gaussian spectrum with half of this bandwidth, it corresponds to a temporal high harmonic pulse with 480 as (FWHM). However, analogously to atomic $\mathrm{HHG}$, the generation process gives an intrinsic temporal chirp to the harmonics that prevents the generation of transform limited pulses (unless the temporal chirp is compensated ex-situ). The chirp arises from the variation in the emission time of different harmonic orders [32]:

$$
C=\frac{d t}{d(N \omega)}
$$

with harmonic order $N(t)$ a function of the return (emission) time. In Fig. 9(a) this relation is depicted by the full blue line for $F_{0}=0.0045$, which is identical to the white line of Fig. 6 . The dashed lines represent the first derivatives of harmonic order with respect to time for the short and long trajectory sides of the curve. The chirp for the long and short trajectories amount to -132 and +288 as/eV respectively. These are larger than those obtained in gases, which typically are $\sim 10 \mathrm{as} / \mathrm{eV}$. The smaller chirp can be attributed to two orders of magnitude higher laser intensity typically used in atomic HHG. As a result, the bandwidth of the harmonic spectrum is much wider resulting in a smaller shift of emission time per harmonic order.

Besides the laser parameters, the shape of the band also affects the attochirp. This is seen by comparing chirps in $\mathrm{ZnO}$ and in atomic gases for field strengths that yield the same cutoff harmonic (at the same fundamental frequency). To achieve that, a field strength of $F_{0}=0.0087\left(F_{0}=F_{\mathrm{v}}\right.$ in a gas) must be chosen for the atomic case. Further, the bandgap $E_{\mathrm{g}}$ is set equal to the atomic ionization potential. The atomic emission times and chirps are the green solid and dashed curves respectively. Whereas the atomic chirp of the short trajectory is $290 \mathrm{as} / \mathrm{eV}$ (comparable to the one from $\mathrm{ZnO}$ ), the chirp of the long trajectory is $-247 \mathrm{as} / \mathrm{eV}$, almost twice the one from $\mathrm{ZnO}$. In atomic gases, due to propagation effects, usually the short trajectories 
are dominant. As was shown above, the presence of dephasing also favours the short over the long trajectories. As a result, the dependence of the long trajectory attochirp on the band structure should not affect experiments.

The pulse generated by the short trajectories has a chirp of $288 \mathrm{as} / \mathrm{eV}$ which corresponds to a group delay dispersion (GDD) equal to $0.19 \mathrm{fs}^{2}$. The conversion is:

$$
G D D\left\{\mathrm{as}^{2}\right\}=\hbar\{\mathrm{eV} \cdot \mathrm{as}\} \times C\{\mathrm{as} / \mathrm{eV}\} .
$$

In the presence of a linear chirp a transform limited pulse of duration $\tau_{t l}$ (FWHM) is broadened to a duration [33] of,

$$
\tau_{c}=\tau_{t l} \sqrt{1+\frac{(4 \ln 2)^{2} G D D^{2}}{\tau_{t l}^{4}}} .
$$

The shorter the pulse and the wider the spectrum, the more severe it is affected by the chirp. As a result, as long as the chirp cannot be compensated, there exists an optimum bandwidth that generates the shortest pulse. The duration of the high-harmonic pulse as a function of its bandwidth is shown in Fig. 9(b). The shortest possible pulse with the given chirp is achieved for $2.5 \mathrm{eV}$ bandwidth and lasts $1.02 \mathrm{fs}$ (transform limit is $0.73 \mathrm{fs}$ ). So far, our estimate has not taken into account how the harmonic intensity changes with harmonic order, which determines the actual bandwidth achievable in experiment. First experiments have shown a strong drop of harmonic intensity with order [9]. This suggests that multi-eV bandwidths are hard to achieve in $\mathrm{ZnO}$, however a $\sim 2.5 \mathrm{eV} \mathrm{FWHM} \mathrm{seems} \mathrm{possible} \mathrm{corresponding}$ to the optimum bandwidth in Fig. 9(b). Therefore, by optimizing material parameters and by going to wider bandgap materials, it seems feasible to achieve sub-fs high-harmonic pulses from solids. Wider bandwidths can also be achieved by excitation to higher lying conduction bands [34] or lower lying valence bands. For the electron, a higher conduction band can be accessed through tunnelling when it reaches the edge of the Brillouin Zone. The probability of such process depends on the bandgap between the two conduction bands and on the field amplitude at this time. For some crystal structures, the bandgap at the edge of the BZ vanishes along some directions. Therefore, by properly choosing the crystal and its orientation, excitation to higher bands is possible.

\section{CONCLUSION}

Our analysis has revealed that the current created through the buildup of interband polarization between valence and conduction band during electron-hole recollision is the dominant mechanism for HHG in solids for excitation by mid-IR lasers. By using quasi-classical trajectory analysis, a simple classical three step model can be developed which is similar to the three step model of HHG in atomic gases. The similarity of HHG in the gas and in the condensed matter phase has opened a path to applying attosecond technology developed for atomic gases to solids. The simple picture outlined in this article is intended to serve as a help and guideline facilitating this process.

Our model presents a very simple approximation to the complex processes that can occur in solids. The next steps for a more complete description of HHG in solids are clear. Sin- gle electron-hole pairs interact through the Coulomb potential, which is neglected in our model. We expect that, in particular during ionization and during recombination, electron-hole interaction will result in corrections. On the one hand the Coulomb potential is expected to play a stronger role than in atomic systems, as electrons and holes are lighter in solids. Also, due to lower laser intensities, the recollision velocities are smaller. These effects are mitigated by many-body screening weakening the electron-hole Coulomb interaction, but overall we expect Coulomb interaction to play a more important role in solids than in atomic gases. An even more complete approach might become necessary at higher laser intensities, where two- and multi-exciton effects will become relevant. Further, structural properties of solids are encoded in the transition dipole moment, whose $\mathbf{k}$-dependence has only been approximated around the $\Gamma$ point in our investigation. In order to identify how the structure of specific solids manifests in harmonic spectra, more complete models for the transition dipole have to be used. Finally, we use a band structure which neglects coupling between coordinate axis, i.e. mixed derivatives of the bandgap are zero. As a result the tensor properties of electron and hole mass are reduced to a diagonal matrix. In solids with non-negligible mixed derivatives, the laser field will also cause dynamics along the other coordinate axes, which can affect properties, such as the dependence of HHG on the ellipticity of the driving laser polarization.

\section{REFERENCES}

[1] P. B. Corkum and F. Krausz, "Attosecond science," Nature Phys., vol. 3, pp. 381-387, 2007.

[2] O. Smirnova et al., "High harmonics interferometry of multi-electron dynamics in molecules," Nature, vol. 460, pp. 972-977, 2009.

[3] J. Itatani et al., "Tomographic imaging of molecular orbitals," Nature, vol. 432, pp. 867-871, 2004.

[4] H. J. Wörner et al., "Following a chemical reaction using high-harmonic interferometry," Nature, vol. 466, pp. 604-607, 2010.

[5] I. J. Sola et al., "Controlling attosecond electron dynamics by phasestabilized polarization gating," Nature Phys., vol. 2, pp. 319-322, 2006.

[6] K. Zhao et al., "Tailoring a 67 attosecond pulse through advantageous phase-mismatch," Opt. Lett., vol. 37.18, pp. 3891-3893, 2012.

[7] C. Vozzi et al., "Generalized molecular orbital tomography," Nature Phys., vol. 7, pp. 822-826, 2011.

[8] A. Ravasio et al., "Single-shot diffractive imaging with a table-top femtosecond soft X-ray laser-harmonics source," Phys. Rev. Lett., vol. 103, pp. 028104, 2009.

[9] S. Ghimire et al., "Observation of high-order harmonic generation in a bulk crystal," Nature Phys., vol. 7, pp. 138-141, 2011.

[10] O. Schubert et al., "Sub-cycle control of terahertz high-harmonic generation by dynamical Bloch oscillations," Nature Photon., vol. 8, pp. 119-123, 2014.

[11] R. W. Boyd, "The nonlinear optical susceptibility," in Nonlinear Optics, 2nd ed. New York, NY, USA: Academic, 2003, ch. 1.

[12] F. Brunel, "Harmonic generation due to plasma effects in a gas undergoing multiphoton ionization in the high-intensity limit," J. Opt. Soc. Amer. B, vol. 7, pp. 521-526, 1990.

[13] D. Golde, and T. Meier, and S. W. Koch, "High harmonics generated in semiconductor nanostructures by the coupled dynamics of optical interand intraband excitations," Phys. Rev. B, vol. 77, pp. 075330, 2008.

[14] G. Vampa, et al., "Theoretical analysis of high harmonic generation in solids," Phys. Rev. Lett., vol. 113, pp. 073901, 2014.

[15] B. Zaks, R. B. Liu, and M. S. Sherwin, "Experimental observation of electron-hole recollisions," Nature, vol. 483, pp. 580-583, 2012.

[16] M. Krüger, M. Schenk, and P. Hommelhoff, "Attosecond control of electrons emitted from a nanoscale metal tip," Nature, vol. 475, pp. 78-81, 2011.

[17] E. Mendez and G. Bastard, "Wannier-Stark ladders and Bloch oscillations in superlattices," Phys. Today, vol. 46, pp. 34-42, 1993. 
[18] D. Golde, M. Kira, T. Meier, and S. W. Koch, "Microscopic theory of the extremely nonlinear terahertz response of semiconductors," Phys. Status Solidi, vol. 248, pp. 863-866, 2011.

[19] F. Kemper, B. Moritz, J. K. Freericks, and T. P. Devereaux, "Theoretical description of high-order harmonic generation in solids," New J. Phys. vol. 15, p. 023003, 2013.

[20] K. A. Pronin, A. D. Bandrauk, and A. A. Ovchinnikov, "Harmonic generation by a one-dimensional conductor: Exact results," Phys. Rev. B, vol. 50, pp. 3473, 1994.

[21] S. Ghimire, et al., "Generation and propagation of high-order harmonics in crystals," Phys. Rev. A, vol. 85, pp. 043836, 2012.

[22] P. G. Hawkins and M. Yu. Ivanov, "Role of subcycle transition dynamics in high-order-harmonic generation in periodic structures," Phys. Rev. A, vol. 87, p. 063842, 2013.

[23] T. Higuchi, M. I. Stockman, and P. Hommelhoff, "Strong-field perspective on high-harmonic radiation from bulk solids," Phys. Rev. Lett., vol. 113 , p. 213901, 2014

[24] M. Goano, F. Bertazzi, M. Penna, and E. Bellotti, "Electronic structure of wurtzite $\mathrm{ZnO}$ : Nonlocal pseudopotential and ab initio calculations," $J$. App. Phys., vol. 102, p. 083709, 2007.

[25] L. Keldysh, "Ionization in the field of a strong electromagnetic wave," Sov. Phys. JETP, vol. 20, pp. 1307-1314, 1965.

[26] J. A. Hostetter, J. L. Tate, K. J. Schafer, and M. B. Gaarde, "Semiclassical approaches to below-threshold harmonics," Phys. Rev. A, vol. 82 pp. 023401, 2010

[27] H. Soifer et al., "Near-threshold high-order harmonic spectroscopy with aligned molecules," Phys. Rev. Lett., vol. 105, pp. 143904, 2010.

[28] O. Smirnova and M. Ivanov, "Multielectron high-harmonic generation: simple man on a complex plane," in Attosecond XUV Spectroscopy: Ultrafast Dynamics and Spectroscopy, 1st ed. New York, NY, USA: Wiley, 2014, chap. 7, sec. 4, pp. 209-221.

[29] G. Vampa et al., "Semi-classical analysis of high harmonic generation in bulk crystals," Phys. Rev. B, vol. 91, p. 064302, 2015.

[30] P. B. Corkum, N. H. Burnett, and M. Yu Ivanov, "Subfemtosecond pulses," Opt. Lett., vol. 19, pp. 1870-1872, 1994.

[31] K. T. Kim, et al., "Photonic streaking of attosecond pulse trains," Nature Photon., vol. 7, pp. 651-656, 2013.

[32] Z. Chang, "Semiclassical model," in Fundamentals of Attosecond Optics, 1st ed. New York, NY, USA: Taylor \& Francis, 2011, ch. 4, sec. 4.1.4., pp. 172-175.

[33] Z. Chang, "Quest for attosecond optical pulses," in Fundamentals of At tosecond Optics, 1st ed. New York, NY, USA: Taylor \& Francis, 2011, ch. 1 , sec. 1.2.2.8, pp. 23.

[34] P. G. Hawkins, M. Yu Ivanov, and V. S. Yakovlev, "The effect of multiple conduction bands on high harmonic emission from dielectrics," Phys. Rev A, vol. 91, art. no. 013405, 2015.
Giulio Vampa received the B.Sc. degree in physics in 2008 and the M.Sc. degree in solid state physics in 2010 from the University of Trieste, Trieste, Italy. He is currently working toward the $\mathrm{Ph}$.D. degree at the University of Ottawa, Ottawa, $\mathrm{ON}$, Canada. His research interest is in strong field interactions with condensed matter. Mr. Vampa has been a Student Member of The Optical Society of America since 2011

Chris McDonald received the doctorate degree in physics from the University of Ottawa, Ottawa, ON, Canada, in 2013, where he is currently a Postdoctoral Fellow. His current research is focused on light-matter interaction.

Alec Fraser has been working toward the Bachelor degree (with Hons.) in mathematics and physics in McGill University Montreal, QC, Canada, since 2012. He is currently studying physics on exchange at École Polytechnique Fédérale de Lausanne, Lausanne, Switzerland.

In 2014, he was a Research Assistant at the Department of Physics, University of Ottawa, Ottawa, ON, Canada. His research interests include nonlinear optics, quantum cryptography and quantum computing.

Mr. Frasers received the McGill University Mobility Award and École Polytechnique Fédérale de Lausanne exchange scholarship.

Thomas Brabec received the M.Eng. degree in 1988 and the Ph.D. degree in 1992 in physics engineering from the Vienna University of Technology, Vienna, Austria.

From 1992 to 1998, he was Research Associate in the Institute of Photonics, Vienna University of Technology. After his Habilitation in 1998, he became an Associate Professor at the Vienna University of Technology. In 2002, he became a Professor in the Department of Physics, University of Ottawa, Ottawa, ON, Canada, and the Canada Research Chair in ultrafast photonics. His research is focused on light-matter interaction, ultrashort pulse physics, and dynamics in many-body systems.

Dr. Brabec received the Austrian START Fellowship in 2000, Canada Research Chair in 2002, and a Steacie Memorial Fellowship in 2004. 